Mathematical Modelling and Analysis

Volume 16 Number 3, September 2011, 376-389

Doi:10.3846/13926292.2011.601769

(C) Vilnius Gediminas Technical University, 2011
Publisher: Taylor\&Francis and VGTU

www.tandfonline.com/loi/tmma20

Online ISSN: 1648-3510

Print ISSN: 1392-6292

\title{
Global Stability of a Three-Species Food-Chain Model with Diffusion and Nonlocal Delays*
}

\section{Xiao Zhang, Rui $\mathrm{Xu}$ and Zhe Li}

\author{
Institute of Applied Mathematics, Shijiazhuang Mechanical Engineering \\ College \\ Shijiazhuang 050003, PR China \\ E-mail: xzhang_82@163.com \\ E-mail(corresp.): rxu88@yahoo.com.cn
}

Received October 13, 2009; revised June 16, 2011; published online August 1, 2011

\begin{abstract}
In this paper, a three species reaction-diffusion food-chain system with nonlocal delays is investigated. Sufficient conditions are derived for the global stability of a positive steady state and boundary steady states of the system by using the energy function method. Numerical simulations are carried out to illustrate the theoretical results.
\end{abstract}

Keywords: food-chain model, reaction-diffusion, nonlocal delay, global stability.

AMS Subject Classification: 35B35; 92D25.

\section{Introduction}

The classical Lotka-Volterra type systems are very important in the models of multi-species populations interactions. Recently, three-species food chain models have been studied by many authors (see, for example, $[3,8,9,11,15$, 16]). In [8], Lin studied the following three-species food-chain system with time delays

$$
\begin{aligned}
& \frac{\partial u_{1}}{\partial t}=d_{1} \frac{\partial^{2} u_{1}}{\partial x^{2}}+u_{1}\left(r_{1}-a_{11} u_{1}-a_{12} u_{2}\left(x, t-\tau_{2}\right)\right) \\
& \frac{\partial u_{2}}{\partial t}=d_{2} \frac{\partial^{2} u_{2}}{\partial x^{2}}+u_{2}\left(-r_{2}+a_{21} u_{1}\left(x, t-\tau_{1}\right)-a_{22} u_{2}-a_{23} u_{3}\left(x, t-\tau_{3}\right)\right), \\
& \frac{\partial u_{3}}{\partial t}=d_{3} \frac{\partial^{2} u_{3}}{\partial x^{2}}+u_{3}\left(-r_{3}+a_{32} u_{2}\left(x, t-\tau_{2}\right)-a_{33} u_{3}\right) \\
& \frac{\partial u_{1}}{\partial n}=\frac{\partial u_{2}}{\partial n}=\frac{\partial u_{3}}{\partial n}=0, \quad(x, t) \in \partial \Omega \times(0,+\infty), \\
& u_{i}(x, \theta)=\phi_{i}(x, \theta) \geq 0 \quad(i=1,2,3), \quad(x, \theta) \in \Omega \times\left[-\tau_{i}, 0\right]
\end{aligned}
$$

* This work was supported by the National Natural Science Foundation of China (No. 11071254). 
where $u_{1}(x, t), u_{2}(x, t)$ and $u_{3}(x, t)$ represent the densities of prey, predator and top predator populations at location $x$ and time $t$, respectively. In [8], the author considered the asymptotic behavior of solution of system (1.1), by using the method of upper and lower solutions.

We note that the time delays of system (1.1) are space-independent. However, in many realistic ecological models, any delays should be spatially inhomogeneous, that is, the delays affect both the temporal and spatial variables. This is due to the fact that any given individual may not necessarily have been at the same spatial location at previous times. Such delays are called spatio-temporal delays or nonlocal delays. The effect of nonlocal delays on the dynamics of ecological models has been taken into account in several papers (see, for example, $[1,2,4,6,5,7,10,13,12,14,17,18,19])$. In [6], Gourley and So introduced a nonlocal delay with the form

$$
\int_{-\infty}^{t} \int_{0}^{\pi} G(x, y, t-s) k(t-s) u(y, s) d y d s
$$

where $G(x, y, t)=\frac{1}{\pi}+\frac{2}{\pi} \sum_{n=1}^{\infty} e^{-d n^{2} t} \cos n x \cos n y$ is solution of

$$
\frac{\partial G}{\partial t}=d \frac{\partial^{2} G}{\partial x^{2}}
$$

subject to $G(x, y, 0)=\delta(x-y)$, the function $k(t)$ is called the delay kernel and satisfies $k(t) \geq 0$ for all $t \geq 0$ together with the normalization condition $\int_{0}^{+\infty} k(t) d t=1$.

Motivated by the work of Lin [8] and Gourley and So [6], in this paper, we study the following reaction-diffusion food-chain model with nonlocal delays

$$
\begin{aligned}
\frac{\partial u_{1}}{\partial t}= & d_{1} \frac{\partial^{2} u_{1}}{\partial x^{2}}+u_{1}\left(r_{1}-a_{11} u_{1}-a_{12} u_{2}\right) \\
\frac{\partial u_{2}}{\partial t}= & d_{2} \frac{\partial^{2} u_{2}}{\partial x^{2}}+u_{2}\left(-r_{2}+a_{21} \int_{-\infty}^{t} \int_{0}^{\pi} G_{1}(x, y, t-s) k_{1}(t-s) u_{1}(y, s) d y d s\right. \\
& \left.-a_{22} u_{2}-a_{23} u_{3}\right) \\
\frac{\partial u_{3}}{\partial t}= & d_{3} \frac{\partial^{2} u_{3}}{\partial x^{2}}+u_{3}\left(-r_{3}+a_{32} \int_{-\infty}^{t} \int_{0}^{\pi} G_{2}(x, y, t-s) k_{2}(t-s) u_{2}(y, s) d y d s\right. \\
& \left.-a_{33} u_{3}\right)
\end{aligned}
$$

for $t>0, x \in(0, \pi)$, with homogeneous Neumann boundary conditions

$$
\frac{\partial u_{1}}{\partial n}=\frac{\partial u_{2}}{\partial n}=\frac{\partial u_{3}}{\partial n}=0, \quad x=0, \pi
$$

and initial conditions

$$
u_{i}(x, \theta)=\phi_{i}(x, \theta) \geq 0 \quad(i=1,2,3), \quad(x, \theta) \in[0, \pi] \times(-\infty, 0],
$$

where

$$
G_{i}(x, y, t)=\frac{1}{\pi}+\frac{2}{\pi} \sum_{n=1}^{\infty} e^{-d_{i} n^{2} t} \cos n x \cos n y, \quad i=1,2
$$


$G_{i}$ is the weight function describing the distribution at past times of the individual of the species $u_{i}$ who is at position $x$ and time $t$. We recall that in system $(1.2), u_{1}(x, t), u_{2}(x, t)$ and $u_{3}(x, t)$ represent the densities of prey, predator and top predator populations at location $x$ and time $t$, respectively. All the parameters in system (1.2) are positive constants.

This paper is organized as follows. In Section 2, we introduce some notations and several lemmas which will be essential to our proofs. In Section 3, we discuss the global stability of steady states for system (1.2). Finally, some numerical simulations are given to illustrate the main theoretical results.

\section{Preliminaries}

In this section, we introduce some notations and several results which will be useful in next section.

Let $R=(-\infty,+\infty), \Omega=(0, \pi)$. For $1 \leq p \leq \infty$, let $L^{p}(\Omega)$ denote the Banach space of measurable functions $u$ on $\Omega$ satisfying

$$
\|u\|_{p}=\left\{\begin{array}{l}
\left(\int_{\Omega}|u(x)|^{p} d x\right)^{1 / p}<\infty \quad \text { if } 1 \leq p<\infty \\
e s s \sup _{x \in \Omega}|u(x)|<\infty \quad \text { if } p=\infty
\end{array}\right.
$$

In particular, if $p=2, L^{2}(\Omega)$ becomes a Hilbert space with usual inner product $\langle\cdot, \cdot\rangle$ and $\|\cdot\|_{2}^{2}=\langle\cdot, \cdot\rangle$. Let $\|\cdot\|_{T}$ denote a norm in $L^{2}\left((0, T) ; L^{2}(\Omega, R)\right)$, i.e.,

$$
\|\cdot\|_{T}=\left(\int_{0}^{T}\|u(s)\|_{2}^{2} d s\right)^{\frac{1}{2}} .
$$

Let $\left(u_{1}(t, x), u_{2}(t, x), u_{3}(t, x)\right)$ be a solution of system (1.2). Then by a comparison theorem we have the following result.

Lemma 1. $\left(u_{1}(t, x), u_{2}(t, x), u_{3}(t, x)\right)$ exists globally and satisfies

$$
0 \leq u_{j}(x, t) \leq \max \left\{M_{j}, \sup _{\theta \leq 0}\left\|\phi_{j}(\theta, \cdot)\right\|_{C(\bar{\Omega} ; R)}\right\}, \quad j=1,2,3,
$$

where $M_{1}=r_{1} / a_{11}, M_{2}=\left(a_{21} M_{1}-r_{2}\right) / a_{22}, M_{3}=\left(a_{32} M_{2}-r_{3}\right) / a_{33}$. Also, by the strong maximum principle, if $\phi_{i}(x, 0) \neq 0(i=1,2,3)$, we have $u_{i}(x, t)>$ $0(i=1,2,3)$, for all $x \in \bar{\Omega}$ and $t>0$.

Moreover, we recall (Lemma 2.1 in [6]).

Lemma 2. Let $K(x, y, t)=G(x, y, t) k(t)$, for the term $\int_{-\infty}^{t} \int_{\Omega} K(x, y, t-$ $s) u(y, s) d y d s$, we have the following inequality

$$
\left\|\int_{-\infty}^{t} \int_{\Omega} K(x, y, t-s) u(y, s) d y d s\right\|_{2} \leq \int_{-\infty}^{t} k(t-s)\|u(s)\|_{2} d s
$$

for any function $u(x, t)$ such that $\frac{\partial u}{\partial n}=0$ on $\partial \Omega$. 


\section{Global Stability}

In this section, we study the global stability of a positive steady state and boundary steady states of system (1.2) by using the energy function method.

It is easy to show that system (1.2) has two steady states $E_{0}(0,0,0)$ and $E_{1}\left(r_{1} / a_{11}, 0,0\right)$. Moreover, if the condition (H1) $a_{21} r_{1}>a_{11} r_{2}$ holds, then system (1.2) has another semi-trivial steady state $E_{2}\left(\tilde{u}_{1}, \tilde{u}_{2}, 0\right)$, where

$$
\tilde{u}_{1}=\frac{a_{22} r_{1}+a_{12} r_{2}}{a_{11} a_{22}+a_{12} a_{21}}, \quad \tilde{u}_{2}=\frac{a_{21} r_{1}-a_{11} r_{2}}{a_{11} a_{22}+a_{12} a_{21}} .
$$

Further, if the condition (H2) $a_{21} a_{32} r_{1}-a_{11} a_{32} r_{2}-a_{11} a_{22} r_{3}-a_{12} a_{21} r_{3}>0$ holds, then system (1.2) has a positive steady state $E^{*}\left(u_{1}^{*}, u_{2}^{*}, u_{3}^{*}\right)$, where

$$
\begin{aligned}
u_{1}^{*} & =\frac{a_{22} a_{33} r_{1}+a_{23} a_{32} r_{1}+a_{12} a_{33} r_{2}-a_{12} a_{23} r_{3}}{a_{11} a_{22} a_{33}+a_{11} a_{23} a_{32}+a_{12} a_{21} a_{33}}, \\
u_{2}^{*} & =\frac{a_{21} a_{33} r_{1}-a_{11} a_{33} r_{2}+a_{11} a_{23} r_{3}}{a_{11} a_{22} a_{33}+a_{11} a_{23} a_{32}+a_{12} a_{21} a_{33}}, \\
u_{3}^{*} & =\frac{a_{21} a_{32} r_{1}-a_{11} a_{32} r_{2}-a_{11} a_{22} r_{3}-a_{12} a_{21} r_{3}}{a_{11} a_{22} a_{33}+a_{11} a_{23} a_{32}+a_{12} a_{21} a_{33}} .
\end{aligned}
$$

We are now in a position to state and prove our main result on the global stability of the positive steady state of system (1.2).

Theorem 1. Let $\left(u_{1}(x, t), u_{2}(x, t), u_{3}(x, t)\right)$ be a solution of system (1.2) with boundary conditions (1.3) and initial conditions (1.4). If (H2) holds and $a_{11} a_{22} a_{33}-a_{11} a_{23} a_{32}-a_{12} a_{21} a_{33}>0$, then

$$
\lim _{t \rightarrow \infty}\left(u_{1}(x, t), u_{2}(x, t), u_{3}(x, t)\right)=\left(u_{1}^{*}, u_{2}^{*}, u_{3}^{*}\right) \quad \text { uniformly for } x \in[0, \pi] .
$$

Proof. Define

$$
\begin{aligned}
& E\left(u_{i}\right)=\int_{\Omega}\left(u_{i}-u_{i}^{*}-u_{i}^{*} \ln \frac{u_{i}}{u_{i}^{*}}\right) d x \quad(i=1,2,3), \\
& K_{i}(x, y, t-s)=G_{i}(x, y, t-s) k_{i}(t-s) \quad(i=1,2) .
\end{aligned}
$$

For some constants $\alpha>0, \beta>0$ to be determined later, we have

$$
\begin{aligned}
\frac{d}{d t}\left[E\left(u_{1}\right)+\alpha E\left(u_{2}\right)+\beta E\left(u_{3}\right)\right] \\
=\int_{\Omega} \frac{\partial u_{1}}{\partial t}\left(1-\frac{u_{1}^{*}}{u_{1}}\right) d x+\alpha \int_{\Omega} \frac{\partial u_{2}}{\partial t}\left(1-\frac{u_{2}^{*}}{u_{2}}\right) d x+\beta \int_{\Omega} \frac{\partial u_{3}}{\partial t}\left(1-\frac{u_{3}^{*}}{u_{3}}\right) d x \\
=-d_{1} u_{1}^{*} \int_{\Omega} \frac{\left|\nabla u_{1}\right|^{2}}{u_{1}^{2}} d x-\alpha d_{2} u_{2}^{*} \int_{\Omega} \frac{\left|\nabla u_{2}\right|^{2}}{u_{2}^{2}} d x-\beta d_{3} u_{3}^{*} \int_{\Omega} \frac{\left|\nabla u_{3}\right|^{2}}{u_{3}^{2}} d x \\
\quad-a_{11} \int_{\Omega}\left(u_{1}-u_{1}^{*}\right)^{2} d x-\alpha a_{22} \int_{\Omega}\left(u_{2}-u_{2}^{*}\right)^{2} d x-\beta a_{33} \int_{\Omega}\left(u_{3}-u_{3}^{*}\right)^{2} d x \\
\quad-a_{12} \int_{\Omega}\left(u_{1}-u_{1}^{*}\right)\left(u_{2}-u_{2}^{*}\right) d x-\alpha a_{23} \int_{\Omega}\left(u_{2}-u_{2}^{*}\right)\left(u_{3}-u_{3}^{*}\right) d x
\end{aligned}
$$




$$
\begin{aligned}
& +\alpha a_{21} \int_{\Omega} \int_{-\infty}^{t} \int_{0}^{\pi} K_{1}(x, y, t-s)\left(u_{1}(y, s)-u_{1}^{*}\right) d y d s\left(u_{2}-u_{2}^{*}\right) d x \\
& +\beta a_{32} \int_{\Omega} \int_{-\infty}^{t} \int_{0}^{\pi} K_{2}(x, y, t-s)\left(u_{2}(y, s)-u_{2}^{*}\right) d y d s\left(u_{3}-u_{3}^{*}\right) d x .
\end{aligned}
$$

Using Young's inequality, we have that

$$
\begin{aligned}
\frac{d}{d t}[E & \left.\left(u_{1}\right)+\alpha E\left(u_{2}\right)+\beta E\left(u_{3}\right)\right] \\
\leq & -d_{1} u_{1}^{*} \int_{\Omega} \frac{\left|\nabla u_{1}\right|^{2}}{u_{1}^{2}} d x-\alpha d_{2} u_{2}^{*} \int_{\Omega} \frac{\left|\nabla u_{2}\right|^{2}}{u_{2}^{2}} d x-\beta d_{3} u_{3}^{*} \int_{\Omega} \frac{\left|\nabla u_{3}\right|^{2}}{u_{3}^{2}} d x \\
& -\left(a_{11}-\frac{a_{12} \eta_{1}}{2}\right) \int_{\Omega}\left(u_{1}-u_{1}^{*}\right)^{2} d x-\left(\alpha a_{22}-\frac{a_{12}}{2 \eta_{1}}-\frac{\alpha a_{23} \eta_{2}}{2}\right) \\
& \times \int_{\Omega}\left(u_{2}-u_{2}^{*}\right)^{2} d x-\left(\beta a_{33}-\frac{\alpha a_{23}}{2 \eta_{2}}\right) \int_{\Omega}\left(u_{3}-u_{3}^{*}\right)^{2} d x \\
& +\alpha a_{21} \int_{\Omega} \int_{-\infty}^{t} \int_{0}^{\pi} K_{1}(x, y, t-s)\left(u_{1}(y, s)-u_{1}^{*}\right) d y d s\left(u_{2}-u_{2}^{*}\right) d x \\
& +\beta a_{32} \int_{\Omega} \int_{-\infty}^{t} \int_{0}^{\pi} K_{2}(x, y, t-s)\left(u_{2}(y, s)-u_{2}^{*}\right) d y d s\left(u_{3}-u_{3}^{*}\right) d x,
\end{aligned}
$$

for any $\eta_{1}, \eta_{2}>0$. It follows from (3.4) that

$$
\begin{aligned}
\frac{d}{d t}[ & \left.E\left(u_{1}\right)+\alpha E\left(u_{2}\right)+\beta E\left(u_{3}\right)\right]+d_{1} u_{1}^{*} \int_{\Omega} \frac{\left|\nabla u_{1}\right|^{2}}{u_{1}^{2}} d x+\alpha d_{2} u_{2}^{*} \int_{\Omega} \frac{\left|\nabla u_{2}\right|^{2}}{u_{2}^{2}} d x \\
& +\beta d_{3} u_{3}^{*} \int_{\Omega} \frac{\left|\nabla u_{3}\right|^{2}}{u_{3}^{2}} d x+\left(a_{11}-\frac{a_{12} \eta_{1}}{2}\right)\left\|u_{1}-u_{1}^{*}\right\|_{2}^{2} \\
& +\left(\alpha a_{22}-\frac{a_{12}}{2 \eta_{1}}-\frac{\alpha a_{23} \eta_{2}}{2}\right)\left\|u_{2}-u_{2}^{*}\right\|_{2}^{2}+\left(\beta a_{33}-\frac{\alpha a_{23}}{2 \eta_{2}}\right)\left\|u_{3}-u_{3}^{*}\right\|_{2}^{2} \\
\leq & \alpha a_{21}\left\langle\int_{-\infty}^{t} \int_{0}^{\pi} K_{1}(x, y, t-s)\left(u_{1}(y, s)-u_{1}^{*}\right) d y d s, u_{2}-u_{2}^{*}\right\rangle \\
& +\beta a_{32}\left\langle\int_{-\infty}^{t} \int_{0}^{\pi} K_{2}(x, y, t-s)\left(u_{2}(y, s)-u_{2}^{*}\right) d y d s, u_{3}-u_{3}^{*}\right\rangle .
\end{aligned}
$$

By Lemma 2, for $i=1,2$, we have that

$$
\begin{aligned}
& \left\|\int_{-\infty}^{t} \int_{0}^{\pi} K_{i}(x, y, t-s)\left(u_{i}(y, s)-u_{i}^{*}\right) d y d s\right\|_{2} \\
& \quad \leq \int_{-\infty}^{t} k_{i}(t-s)\left\|u_{i}(s)-u_{i}^{*}\right\|_{2} d s \\
& \quad \leq \sup _{s \leq 0}\left\|u_{i}(s)-u_{i}^{*}\right\|_{2} \int_{t}^{\infty} k_{i}(s) d s+\int_{0}^{t} k_{i}(t-s)\left\|u_{i}(s)-u_{i}^{*}\right\|_{2} d s .
\end{aligned}
$$


For any $T>0$, we have

$$
\begin{aligned}
& \left|\int_{0}^{T}\left\langle\int_{-\infty}^{t} \int_{0}^{\pi} K_{1}(x, y, t-s)\left(u_{1}(y, s)-u_{1}^{*}\right) d y d s, u_{2}(t)-u_{2}^{*}\right\rangle d t\right| \\
& \quad \leq \int_{0}^{T}\left\|u_{2}(t)-u_{2}^{*}\right\|_{2}\left\|\int_{-\infty}^{t} \int_{0}^{\pi} K_{1}(x, y, t-s)\left(u_{1}(y, s)-u_{1}^{*}\right) d y d s\right\|_{2} d t \\
& \quad \leq \sup _{s \leq 0}\left\|u_{1}(s)-u_{1}^{*}\right\|_{2} \sup _{0 \leq t \leq T}\left\|u_{2}(s)-u_{2}^{*}\right\|_{2} \int_{0}^{\infty} s k_{1}(s) d s \\
& \quad+\int_{0}^{T}\left\|u_{2}(t)-u_{2}^{*}\right\|_{2} \int_{0}^{t} k_{1}(t-s)\left\|u_{1}(s)-u_{1}^{*}\right\|_{2} d s d t .
\end{aligned}
$$

We estimate the second term in (3.7) that

$$
\begin{aligned}
& \int_{0}^{T}\left\|u_{2}(t)-u_{2}^{*}\right\|_{2} \int_{0}^{t} k_{1}(t-s)\left\|u_{1}(s)-u_{1}^{*}\right\|_{2} d s d t \\
& \quad \leq\left\|u_{2}-u_{2}^{*}\right\|_{T}\left(\int_{0}^{T}\left(\int_{0}^{t} k_{1}(t-s)\left\|u_{1}(s)-u_{1}^{*}\right\|_{2} d s\right)^{2} d t\right)^{1 / 2} \\
& \quad \leq\left\|u_{2}-u_{2}^{*}\right\|_{T}\left(\int_{0}^{T}\left(\int_{0}^{t} k_{1}(t-s) d s\right) \int_{0}^{t} k_{1}(t-s)\left\|u_{1}(s)-u_{1}^{*}\right\|_{2}^{2} d s d t\right)^{1 / 2} \\
& \quad \leq\left\|u_{2}-u_{2}^{*}\right\|_{T}\left(\int_{0}^{T} \int_{0}^{t} k_{1}(t-s)\left\|u_{1}(s)-u_{1}^{*}\right\|_{2}^{2} d s d t\right)^{1 / 2} \\
& \quad=\left\|u_{2}-u_{2}^{*}\right\|_{T}\left(\int_{0}^{T}\left\|u_{1}(s)-u_{1}^{*}\right\|_{2}^{2} \int_{s}^{T} k_{1}(t-s) d t d s\right)^{1 / 2} \\
& \quad \leq\left\|u_{2}-u_{2}^{*}\right\|_{T}\left\|u_{1}-u_{1}^{*}\right\|_{T},
\end{aligned}
$$

where $\|\cdot\|_{T}$ denotes the norm defined as in (2.2). Therefore, for any $T>0$, we have

$$
\begin{aligned}
& \left|\int_{0}^{T}\left\langle\int_{-\infty}^{t} \int_{0}^{\pi} K_{1}(x, y, t-s)\left(u_{1}(y, s)-u_{1}^{*}\right) d y d s, u_{2}(t)-u_{2}^{*}\right\rangle d t\right| \\
& \quad \leq \sup _{s \leq 0}\left\|u_{1}(s)-u_{1}^{*}\right\|_{2} \sup _{0 \leq t \leq T}\left\|u_{2}(s)-u_{2}^{*}\right\|_{2} \int_{0}^{\infty} s k_{1}(s) d s \\
& \quad+\left\|u_{2}-u_{2}^{*}\right\|_{T}\left\|u_{1}-u_{1}^{*}\right\|_{T} .
\end{aligned}
$$

In a similar way, we have that

$$
\begin{aligned}
& \left|\int_{0}^{T}\left\langle\int_{-\infty}^{t} \int_{0}^{\pi} K_{2}(x, y, t-s)\left(u_{2}(y, s)-u_{2}^{*}\right) d y d s, u_{3}(t)-u_{3}^{*}\right\rangle d t\right| \\
& \quad \leq \sup _{s \leq 0}\left\|u_{2}(s)-u_{2}^{*}\right\|_{2} \sup _{0 \leq t \leq T}\left\|u_{3}(s)-u_{3}^{*}\right\|_{2} \int_{0}^{\infty} s k_{2}(s) d s \\
& \quad+\left\|u_{2}-u_{2}^{*}\right\|_{T}\left\|u_{3}-u_{3}^{*}\right\|_{T} .
\end{aligned}
$$


Integrating (3.5) over $[0, T]$, and noting that $\sup _{0 \leq t \leq T}\left\|u_{1}(s)-u_{1}^{*}\right\|_{2}, \sup _{0 \leq t \leq T} \| u_{2}(s)-$ $u_{2}^{*} \|_{2}$ and $\sup _{0 \leq t \leq T}\left\|u_{3}(s)-u_{3}^{*}\right\|_{2}$ can be bounded independently of $T$ (by Lemma 1 ), we obtain that there exists a positive constant $\mathrm{C}$ independent of $T$ such that

$$
\begin{gathered}
d_{1} u_{1}^{*}\left\|\frac{\nabla u_{1}}{u_{1}}\right\|_{T}^{2}+\alpha d_{2} u_{2}^{*}\left\|\frac{\nabla u_{2}}{u_{2}}\right\|_{T}^{2}+\beta d_{3} u_{3}^{*}\left\|\frac{\nabla u_{3}}{u_{3}}\right\|_{T}^{2}+\left(a_{11}-\frac{a_{12} \eta_{1}}{2}\right)\left\|u_{1}-u_{1}^{*}\right\|_{T}^{2} \\
+\left(\alpha a_{22}-\frac{a_{12}}{2 \eta_{1}}-\frac{\alpha a_{23} \eta_{2}}{2}\right)\left\|u_{2}-u_{2}^{*}\right\|_{T}^{2}+\left(\beta a_{33}-\frac{\alpha a_{23}}{2 \eta_{2}}\right)\left\|u_{3}-u_{3}^{*}\right\|_{T}^{2} \\
\leq C+\alpha a_{21}\left\|u_{2}-u_{2}^{*}\right\|_{T}\left\|u_{1}-u_{1}^{*}\right\|_{T}+\beta a_{32}\left\|u_{2}-u_{2}^{*}\right\|_{T}\left\|u_{3}-u_{3}^{*}\right\|_{T} .
\end{gathered}
$$

By using the Young's inequality, we have

$$
\begin{aligned}
d_{1} u_{1}^{*} & \left\|\frac{\nabla u_{1}}{u_{1}}\right\|_{T}^{2}+\alpha d_{2} u_{2}^{*}\left\|\frac{\nabla u_{2}}{u_{2}}\right\|_{T}^{2}+\beta d_{3} u_{3}^{*}\left\|\frac{\nabla u_{3}}{u_{3}}\right\|_{T}^{2}+\left(a_{11}-\frac{a_{12} \eta_{1}}{2}\right)\left\|u_{1}-u_{1}^{*}\right\|_{T}^{2} \\
& +\left(\alpha a_{22}-\frac{a_{12}}{2 \eta_{1}}-\frac{\alpha a_{23} \eta_{2}}{2}\right)\left\|u_{2}-u_{2}^{*}\right\|_{T}^{2}+\left(\beta a_{33}-\frac{\alpha a_{23}}{2 \eta_{2}}\right)\left\|u_{3}-u_{3}^{*}\right\|_{T}^{2} \\
\leq & C+\alpha a_{21}\left(\frac{\varepsilon_{1}}{2}\left\|u_{1}-u_{1}^{*}\right\|_{T}^{2}+\frac{1}{2 \varepsilon_{1}}\left\|u_{2}-u_{2}^{*}\right\|_{T}^{2}\right) \\
& +\beta a_{32}\left(\frac{\varepsilon_{2}}{2}\left\|u_{2}-u_{2}^{*}\right\|_{T}^{2}+\frac{1}{2 \varepsilon_{2}}\left\|u_{3}-u_{3}^{*}\right\|_{T}^{2}\right),
\end{aligned}
$$

for any $\varepsilon_{1}, \varepsilon_{2}>0$. We choose $\varepsilon_{1}=\eta_{1}=2 a_{11} /\left(a_{12}+\alpha a_{21}\right), \varepsilon_{2}=\eta_{2}=$ $\left(\alpha a_{23}+\beta a_{32}\right) / 2 \beta a_{33}$. Then (3.12) becomes

$$
\begin{aligned}
& d_{1} u_{1}^{*}\left\|\frac{\nabla u_{1}}{u_{1}}\right\|_{T}^{2}+\alpha d_{2} u_{2}^{*}\left\|\frac{\nabla u_{2}}{u_{2}}\right\|_{T}^{2}+\beta d_{3} u_{3}^{*}\left\|\frac{\nabla u_{3}}{u_{3}}\right\|_{T}^{2}+\left(\alpha a_{22}-\frac{a_{12}}{2 \eta_{1}}-\frac{\alpha a_{23} \eta_{2}}{2}\right) \\
& \quad \times\left\|u_{2}-u_{2}^{*}\right\|_{T}^{2} \leq C+\left(\alpha a_{21} \frac{1}{2 \varepsilon_{1}}+\beta a_{32} \frac{\varepsilon_{2}}{2}\right)\left\|u_{2}-u_{2}^{*}\right\|_{T}^{2}
\end{aligned}
$$

If $\alpha a_{22}-\frac{a_{12}}{2 \eta_{1}}-\frac{\alpha a_{23} \eta_{2}}{2}>\alpha a_{21} \frac{1}{2 \varepsilon_{1}}+\beta a_{32} \frac{\varepsilon_{2}}{2}$, from (3.13) we can conclude that

$$
\left\|\frac{\nabla u_{1}}{u_{1}}\right\|_{T} \leq C_{1}, \quad\left\|\frac{\nabla u_{2}}{u_{2}}\right\|_{T} \leq C_{2}, \quad\left\|\frac{\nabla u_{3}}{u_{3}}\right\|_{T} \leq C_{3}, \quad\left\|u_{2}-u_{2}^{*}\right\|_{T} \leq C_{4},
$$

for some constants $C_{i}(i=1,2,3,4)$ independent of $T$.

We can choose $\alpha, \beta>0$ satisfying $\alpha a_{22}-\frac{a_{12}}{2 \eta_{1}}-\frac{\alpha a_{23} \eta_{2}}{2}>\alpha a_{21} \frac{1}{2 \varepsilon_{1}}+\beta a_{32} \frac{\varepsilon_{2}}{2}$. Noting that $\varepsilon_{1}=\eta_{1}=\frac{2 a_{11}}{a_{12}+\alpha a_{21}}, \varepsilon_{2}=\eta_{2}=\frac{\alpha a_{23}+\beta a_{32}}{2 \beta a_{33}}$, we obtain that

$$
\beta^{2} a_{32}^{2}+2 \beta\left(\frac{a_{33}\left(a_{12}+\alpha a_{21}\right)^{2}}{2 a_{11}}-2 \alpha a_{22} a_{33}+\alpha a_{32} a_{23}\right)+\alpha^{2} a_{23}^{2}<0 .
$$

Denote

$$
\begin{aligned}
& \Delta_{1}=4\left(\frac{a_{33}\left(a_{12}+\alpha a_{21}\right)^{2}}{2 a_{11}}-2 \alpha a_{22} a_{33}+\alpha a_{32} a_{23}\right)^{2}-4 a_{32}^{2} \alpha^{2} a_{23}^{2}, \\
& B_{1}=\frac{a_{33}\left(a_{12}+\alpha a_{21}\right)^{2}}{2 a_{11}}-2 \alpha a_{22} a_{33}+\alpha a_{32} a_{23} .
\end{aligned}
$$


If $\Delta_{1}>0, B_{1}<0$, then there is a $\beta>0$ such that the inequality (3.15) holds. For $\Delta_{1}>0, B_{1}<0$, we get that

$$
\begin{aligned}
& a_{33}\left(a_{12}+\alpha a_{21}\right)^{2} / 2 a_{11}-2 \alpha a_{22} a_{33}+2 \alpha a_{32} a_{23}<0 \\
& a_{33}\left(a_{12}+\alpha a_{21}\right)^{2}-4 \alpha a_{11} a_{22} a_{33}+4 \alpha a_{11} a_{32} a_{23}<0 \\
& \alpha^{2} a_{21}^{2} a_{33}+2 \alpha\left(2 a_{11} a_{23} a_{32}-2 a_{11} a_{22} a_{33}+a_{12} a_{21} a_{33}\right)+a_{12}^{2} a_{33}<0 .
\end{aligned}
$$

Denote

$$
\begin{aligned}
\Delta_{2} & =4\left(2 a_{11} a_{23} a_{32}-2 a_{11} a_{22} a_{33}+a_{12} a_{21} a_{33}\right)^{2}-4 a_{21}^{2} a_{33}^{2} a_{12}^{2} \\
& =16\left(a_{11} a_{23} a_{32}-a_{11} a_{22} a_{33}+a_{21} a_{12} a_{33}\right)\left(a_{11} a_{23} a_{32}-a_{11} a_{22} a_{33}\right), \\
B_{2} & =2 a_{11} a_{23} a_{32}-2 a_{11} a_{22} a_{33}+a_{12} a_{21} a_{33} .
\end{aligned}
$$

Noting that $a_{11} a_{22} a_{33}-a_{11} a_{23} a_{32}-a_{12} a_{21} a_{33}>0$, we obtain that $\Delta_{2}>0$, $B_{2}<0$. Then there exists $\alpha>0$ such that (3.16) holds. Therefore, the inequality (3.15) holds. Because of (2.3) we may deduce from (3.14) that, for some constant $C_{5}$ independent of $T$,

$$
\left\|\nabla u_{2}\right\|_{T} \leq C_{5}
$$

Inequalities (3.14) and (3.18) imply that $u_{2}-u_{2}^{*} \in L^{2}\left((0, \infty) ; W^{1,2}(\Omega ; R)\right)$ and thus

$$
\lim _{t \rightarrow \infty}\left\|u_{2}-u_{2}^{*}\right\|_{W^{1,2}}=0
$$

Therefore,

$$
\lim _{t \rightarrow \infty}\left\|u_{2}-u_{2}^{*}\right\|_{C(\bar{\Omega} ; R)}=0 .
$$

In a similar way, we derive that

$$
\lim _{t \rightarrow \infty}\left\|u_{1}-u_{1}^{*}\right\|_{C(\bar{\Omega} ; R)}=0, \quad \lim _{t \rightarrow \infty}\left\|u_{3}-u_{3}^{*}\right\|_{C(\bar{\Omega} ; R)}=0 .
$$

The proof is complete.

Next, we consider the global stability of the boundary steady state of system (1.2).

Theorem 2. Let $\left(u_{1}(x, t), u_{2}(x, t), u_{3}(x, t)\right)$ be a solution of system (1.2) with boundary conditions (1.3) and initial conditions (1.4). If $a_{21} r_{1} \leq a_{11} r_{2}$ and $a_{11} a_{22}>a_{12} a_{21}$, then

$$
\lim _{t \rightarrow \infty}\left(u_{1}(x, t), u_{2}(x, t), u_{3}(x, t)\right)=\left(r_{1} / a_{11}, 0,0\right) \quad \text { uniformly for } x \in[0, \pi] .
$$

Proof. Define

$$
\begin{aligned}
& E\left(u_{1}\right)=\int_{\Omega}\left(u_{1}-\frac{r_{1}}{a_{11}}-\frac{r_{1}}{a_{11}} \ln \frac{a_{11} u_{1}}{r_{1}}\right) d x, \quad F\left(u_{i}\right)=\int_{\Omega} u_{i} d x, \quad i=2,3 \\
& K_{i}(x, y, t-s)=G_{i}(x, y, t-s) k_{i}(t-s) \quad(i=1,2) .
\end{aligned}
$$


For some constants $\alpha, \beta>0$ to be determined later, we have

$$
\begin{aligned}
& \frac{d}{d t}\left[E\left(u_{1}\right)+\alpha F\left(u_{2}\right)+\beta F\left(u_{3}\right)\right] \\
& =\int_{\Omega} \frac{\partial u_{1}}{\partial t}\left(1-\frac{r_{1}}{a_{11} u_{1}}\right) d x+\alpha \int_{\Omega} \frac{\partial u_{2}}{\partial t} d x+\beta \int_{\Omega} \frac{\partial u_{3}}{\partial t} d x \\
& =-\frac{d_{1} r_{1}}{a_{11}} \int_{\Omega} \frac{\left|\nabla u_{1}\right|^{2}}{u_{1}^{2}} d x-a_{11} \int_{\Omega}\left(u_{1}-\frac{r_{1}}{a_{11}}\right)^{2} d x-\alpha a_{22} \int_{\Omega} u_{2}^{2} d x \\
& -\beta a_{33} \int_{\Omega} u_{3}^{2} d x-a_{12} \int_{\Omega}\left(u_{1}-\frac{r_{1}}{a_{11}}\right) u_{2} d x-\alpha \int_{\Omega} r_{2} u_{2} d x-\alpha a_{23} \int_{\Omega} u_{2} u_{3} d x \\
& -\beta \int_{\Omega} r_{3} u_{3} d x+\alpha a_{21} \int_{\Omega}\left(\int_{-\infty}^{t} \int_{0}^{\pi} K_{1}(x, y, t-s) u_{1}(y, s) d y d s\right) u_{2} d x \\
& \quad+\beta a_{32} \int_{\Omega}\left(\int_{-\infty}^{\pi} \int_{0}^{\pi} K_{2}(x, y, t-s) u_{2}(y, s) d y d s\right) u_{3} d x .
\end{aligned}
$$

Noting that $a_{11} r_{2} \geq a_{21} r_{1}$, by using the Young's inequality we have

$$
\begin{aligned}
\frac{d}{d t}[ & \left.E\left(u_{1}\right)+\alpha F\left(u_{2}\right)+\beta F\left(u_{3}\right)\right] \\
\leq & -\frac{r_{1} d_{1}}{a_{11}} \int_{\Omega} \frac{\left|\nabla u_{1}\right|^{2}}{u_{1}^{2}} d x-\left(a_{11}-\frac{a_{12} \eta_{1}}{2}\right) \int_{\Omega}\left(u_{1}-\frac{r_{1}}{a_{11}}\right)^{2} d x \\
& -\left(\alpha a_{22}-\frac{a_{12}}{2 \eta_{1}}\right) \int_{\Omega} u_{2}^{2} d x-\beta a_{33} \int_{\Omega} u_{3}^{2} d x \\
& +\alpha a_{21} \int_{\Omega}\left(\int_{-\infty}^{t} \int_{0}^{\pi} K_{1}(x, y, t-s)\left(u_{1}(y, s)-\frac{r_{1}}{a_{11}}\right) d y d s\right) u_{2} d x \\
& +\beta a_{32} \int_{\Omega}\left(\int_{-\infty}^{t} \int_{0}^{\pi} K_{2}(x, y, t-s) u_{2}(y, s) d y d s\right) u_{3} d x
\end{aligned}
$$

It follows from (3.23) that

$$
\begin{aligned}
& \frac{d}{d t}\left[E\left(u_{1}\right)+\alpha F\left(u_{2}\right)+\beta F\left(u_{3}\right)\right]+\frac{d_{1} r_{1}}{a_{11}} \int_{\Omega} \frac{\left|\nabla u_{1}\right|^{2}}{u_{1}^{2}} d x \\
& \quad+\left(a_{11}-\frac{a_{12} \eta_{1}}{2}\right)\left\|u_{1}-\frac{r_{1}}{a_{11}}\right\|_{2}^{2}+\left(\alpha a_{22}-\frac{a_{12}}{2 \eta_{1}}\right)\left\|u_{2}\right\|_{2}^{2}+\beta a_{33}\left\|u_{3}\right\|_{2}^{2} \\
& \leq \alpha a_{21}\left\langle\int_{-\infty}^{t} \int_{0}^{\pi} K_{1}(x, y, t-s)\left(u_{1}(y, s)-\frac{r_{1}}{a_{11}}\right) d y d s, u_{2}\right\rangle \\
& \quad+\beta a_{32}\left\langle\int_{-\infty}^{t} \int_{0}^{\pi} K_{2}(x, y, t-s) u_{2}(y, s) d y d s, u_{3}\right\rangle .
\end{aligned}
$$

By Lemma 2, we have that

$\left\|\int_{-\infty}^{t} \int_{0}^{\pi} K_{1}(x, y, t-s)\left(u_{1}(y, s)-\frac{r_{1}}{a_{11}}\right) d y d s\right\|_{2} \leq \int_{-\infty}^{t} k_{1}(t-s)\left\|u_{1}(s)-\frac{r_{1}}{a_{11}}\right\|_{2} d s$ 


$$
\leq \sup _{s \leq 0}\left\|u_{1}(s)-\frac{r_{1}}{a_{11}}\right\|_{2} \int_{t}^{\infty} k_{1}(s) d s+\int_{0}^{t} k_{1}(t-s)\left\|u_{1}(s)-\frac{r_{1}}{a_{11}}\right\|_{2} d s .
$$

For any $T>0$, we have

$$
\begin{aligned}
& \left|\int_{0}^{T}\left\langle\int_{-\infty}^{t} \int_{0}^{\pi} K_{1}(x, y, t-s)\left(u_{1}(y, s)-\frac{r_{1}}{a_{11}}\right) d y d s, u_{2}(t)\right\rangle d t\right| \\
& \quad \leq \int_{0}^{T}\left\|u_{2}(t)\right\|_{2}\left\|\int_{-\infty}^{t} \int_{0}^{\pi} K_{1}(x, y, t-s)\left(u_{1}(y, s)-\frac{r_{1}}{a_{11}}\right) d y d s\right\|_{2} d t \\
& \quad \leq \sup _{s \leq 0}\left\|u_{1}(s)-\frac{r_{1}}{a_{11}}\right\|_{2} \sup _{0 \leq t \leq T}\left\|u_{2}(s)\right\|_{2} \int_{0}^{\infty} s k_{1}(s) d s \\
& \quad+\int_{0}^{T}\left\|u_{2}(t)\right\|_{2} \int_{0}^{t} k_{1}(t-s)\left\|u_{1}(s)-\frac{r_{1}}{a_{11}}\right\|_{2} d s d t
\end{aligned}
$$

We estimate the second term in (3.26) that

$$
\begin{aligned}
& \int_{0}^{T}\left\|u_{2}(t)\right\|_{2} \int_{0}^{t} k_{1}(t-s)\left\|u_{1}(s)-\frac{r_{1}}{a_{11}}\right\|_{2} d s d t \\
& \quad \leq\left\|u_{2}\right\|_{T}\left(\int_{0}^{T}\left(\int_{0}^{t} k_{1}(t-s)\left\|u_{1}(s)-\frac{r_{1}}{a_{11}}\right\|_{2} d s\right)^{2} d t\right)^{1 / 2} \\
& \quad \leq\left\|u_{2}\right\|_{T}\left(\int_{0}^{T}\left(\int_{0}^{t} k_{1}(t-s) d s\right) \int_{0}^{t} k_{1}(t-s)\left\|u_{1}(s)-\frac{r_{1}}{a_{11}}\right\|_{2}^{2} d s d t\right)^{1 / 2} \\
& \quad \leq\left\|u_{2}\right\|_{T}\left(\int_{0}^{T} \int_{0}^{t} k_{1}(t-s)\left\|u_{1}(s)-\frac{r_{1}}{a_{11}}\right\|_{2}^{2} d s d t\right)^{1 / 2} \\
& \quad=\left\|u_{2}\right\|_{T}\left(\int_{0}^{T}\left\|u_{1}(s)-\frac{r_{1}}{a_{11}}\right\|_{2}^{2} \int_{s}^{T} k_{1}(t-s) d t d s\right)^{1 / 2} \leq\left\|u_{2}\right\|_{T}\left\|u_{1}-\frac{r_{1}}{a_{11}}\right\|_{T}
\end{aligned}
$$

where $\|\cdot\|_{T}$ denotes the norm defined as in (2.2). Therefore, for any $T>0$, we have

$$
\begin{aligned}
& \left|\int_{0}^{T}\left\langle\int_{-\infty}^{t} \int_{0}^{\pi} K_{1}(x, y, t-s)\left(u_{1}(y, s)-\frac{r_{1}}{a_{11}}\right) d y d s, u_{2}(t)\right\rangle d t\right| \\
& \quad \leq \sup _{s \leq 0}\left\|u_{1}(s)-\frac{r_{1}}{a_{11}}\right\|_{2} \sup _{0 \leq t \leq T}\left\|u_{2}(s)\right\|_{2} \int_{0}^{\infty} s k_{1}(s) d s+\left\|u_{2}\right\|\left\|_{T}\right\| u_{1}-\frac{r_{1}}{a_{11}} \|_{T} .
\end{aligned}
$$

In a similar way, we have that

$$
\begin{aligned}
& \left|\int_{0}^{T}\left\langle\int_{-\infty}^{t} \int_{0}^{\pi} K_{2}(x, y, t-s) u_{2}(y, s) d y d s, u_{3}(t)\right\rangle d t\right| \\
& \quad \leq \sup _{s \leq 0}\left\|u_{2}(s)\right\|_{2} \sup _{0 \leq t \leq T}\left\|u_{3}(s)\right\|_{2} \int_{0}^{\infty} s k_{2}(s) d s+\left\|u_{2}\right\|_{T}\left\|u_{3}\right\|_{T} .
\end{aligned}
$$

Integrating (3.24) over $[0, T]$, and noting that $\sup _{0 \leq t \leq T}\left\|u_{1}-\frac{r_{1}}{a_{11}}\right\|_{2}, \sup _{0 \leq t \leq T}\left\|u_{2}(s)\right\|_{2}$, $\sup \left\|u_{3}(s)\right\|_{2}$ can be bounded independently of $T$ (by Lemma 1 ), we obtain $0 \leq t \leq T$ 
that there exists a positive constant $\mathrm{C}$ independent of $T$ such that

$$
\begin{aligned}
& \frac{r_{1} d_{1}}{a_{11}}\left\|\frac{\nabla u_{1}}{u_{1}}\right\|_{T}^{2}+\left(a_{11}-\frac{a_{12} \eta_{1}}{2}\right)\left\|u_{1}-\frac{r_{1}}{a_{11}}\right\|_{T}^{2}+\left(\alpha a_{22}-\frac{a_{12}}{2 \eta_{1}}\right)\left\|u_{2}\right\|_{T}^{2} \\
& +\beta a_{33}\left\|u_{3}\right\|_{T}^{2} \leq C+\alpha a_{21}\left\|u_{2}\right\|_{T}\left\|u_{1}-\frac{r_{1}}{a_{11}}\right\|_{T}+\beta a_{32}\left\|u_{2}\right\|\left\|_{T}\right\| u_{3} \|_{T} .
\end{aligned}
$$

By using the Young's inequality, we have

$$
\begin{aligned}
& \frac{r_{1} d_{1}}{a_{11}}\left\|\frac{\nabla u_{1}}{u_{1}}\right\|_{T}^{2}+\left(a_{11}-\frac{a_{12} \eta_{1}}{2}\right)\left\|u_{1}-\frac{r_{1}}{a_{11}}\right\|_{T}^{2}+\left(\alpha a_{22}-\frac{a_{12}}{2 \eta_{1}}\right)\left\|u_{2}\right\|_{T}^{2}+\beta a_{33}\left\|u_{3}\right\|_{T}^{2} \\
& \leq C+\alpha a_{21}\left(\frac{\varepsilon_{1}}{2}\left\|u_{1}-\frac{r_{1}}{a_{11}}\right\|_{T}^{2}+\frac{1}{2 \varepsilon_{1}}\left\|u_{2}\right\|_{T}^{2}\right)+\beta a_{32}\left(\frac{\varepsilon_{2}}{2}\left\|u_{2}\right\|_{T}^{2}+\frac{1}{2 \varepsilon_{2}}\left\|u_{3}\right\|_{T}^{2}\right), \quad \text { (3.31) }
\end{aligned}
$$

for any $\varepsilon_{1}, \varepsilon_{2}>0$. We choose $\varepsilon_{1}=\eta_{1}=2 a_{11} / a_{12}+\alpha a_{21}, \varepsilon_{2}=a_{32} / 2 a_{33}$. Then (3.31) becomes

$$
\frac{r_{1} d_{1}}{a_{11}}\left\|\frac{\nabla u_{1}}{u_{1}}\right\|_{T}^{2}+\left(\alpha a_{22}-\frac{a_{12}}{2 \eta_{1}}\right)\left\|u_{2}\right\|_{T}^{2} \leq C+\left(\alpha a_{21} \frac{1}{2 \varepsilon_{1}}+\beta a_{32} \frac{\varepsilon_{2}}{2}\right)\left\|u_{2}\right\|_{T}^{2} .
$$

If $\alpha a_{22}-a_{12} / 2 \eta_{1}>\alpha a_{21} / 2 \varepsilon_{1}+\beta a_{32} \varepsilon_{2} / 2$, from (3.32) we can conclude that

$$
\left\|\frac{\nabla u_{1}}{u_{1}}\right\|_{T} \leq C_{1}, \quad\left\|u_{2}\right\|_{T} \leq C_{4}
$$

for some constants $C_{i}(i=1,2,3,4)$ independent of $T$. We can choose $\alpha, \beta>0$ satisfying $\alpha a_{22}-a_{12} / 2 \eta_{1}>\alpha a_{21} / 2 \varepsilon_{1}+\beta a_{32} \varepsilon_{2} / 2$. Noting that $\varepsilon_{1}=\eta_{1}=$ $2 a_{11} / a_{12}+\alpha a_{21}, \varepsilon_{2}=a_{32} / 2 a_{33}$, we obtain that

$$
\alpha^{2} a_{21}^{2} a_{33}+2 a_{33} \alpha\left(a_{12} a_{21}-2 a_{11} a_{22}\right)+\beta a_{11} a_{32}^{2}+a_{33} a_{12}^{2}<0 .
$$

Denote $\Delta_{1}=4 a_{33}^{2}\left(a_{12} a_{21}-2 a_{11} a_{22}\right)^{2}-4 a_{21}^{2} a_{33}\left(\beta a_{11} a_{32}^{2}+a_{33} a_{12}^{2}\right)$. Noting that $a_{11} a_{22}>a_{12} a_{21}$, we know that if $\Delta_{1}>0$, there exists an $\alpha>0$ such that inequality (3.34) holds. For $\Delta_{1}>0$, we get that

$$
4 a_{11} a_{22} a_{33}^{2}\left(a_{11} a_{22}-a_{12} a_{21}\right)>\beta a_{11} a_{21}^{2} a_{32}^{2} a_{33} .
$$

So we can choose $0<\beta<4 a_{22} a_{33}\left(a_{11} a_{22}-a_{12} a_{21}\right) / a_{21}^{2} a_{32}^{2}$. Therefore, inequality (3.34) holds. Hence, $\lim _{t \rightarrow \infty}\left\|u_{2}\right\|_{C(\bar{\Omega} ; R)}=0$.

In a similar way, we derive that

$$
\lim _{t \rightarrow \infty}\left\|u_{1}-r_{1} / a_{11}\right\|_{C(\bar{\Omega} ; R)}=0, \quad \lim _{t \rightarrow \infty}\left\|u_{3}\right\|_{C(\bar{\Omega} ; R)}=0 .
$$

The proof is complete.

Using a similar argument, we can also prove the following results.

Theorem 3. Let $\left(u_{1}(x, t), u_{2}(x, t), u_{3}(x, t)\right)$ be a solution of system (1.2) with boundary conditions (1.3) and initial conditions (1.4). If

$$
\begin{aligned}
& a_{11} a_{22} a_{33}-a_{11} a_{23} a_{32}-a_{12} a_{21} a_{33}>0, \\
& a_{21} a_{32} r_{1}-a_{11} a_{32} r_{2}-a_{11} a_{22} r_{3}-a_{12} a_{21} r_{3} \leq 0
\end{aligned}
$$

hold and (H1) is valid, then $\lim _{t \rightarrow \infty}\left(u_{1}(x, t), u_{2}(x, t), u_{3}(x, t)\right)=\left(\tilde{u}_{1}, \tilde{u}_{2}, 0\right)$ uniformly for $x \in[0, \pi]$. 


\section{Numerical Simulations}

In this section, we present some numerical simulations to illustrate the results in Section 3. In system (1.2), we let $f_{i}(t)=\frac{1}{\tau_{i}} \exp \left(-t / \tau_{i}\right), i=1,2$.

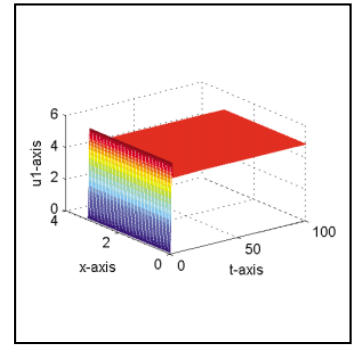

(a)

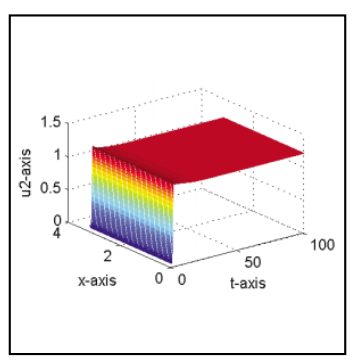

(b)

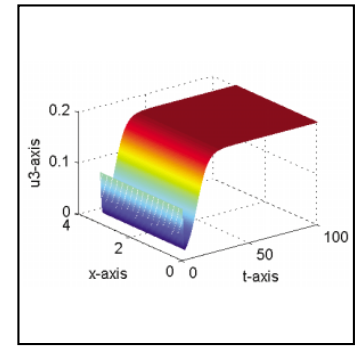

(c)

Figure 1. The time dependent solution found by numerical integration with $r_{1}=6$, $r_{2}=r_{3}=1, a_{11}=a_{12}=a_{21}=1, a_{22}=3, a_{23}=a_{32}=a_{33}=1, d_{1}=1, d_{2}=2, d_{3}=1$, $\tau_{1}=\tau_{2}=0.1 ; \phi_{i}(x, 0)=0.1(i=1,2,3) ; \partial u_{i} / \partial x=0(i=1,2,3), t \geq 0, x=0, \pi$.

Example 1. In system (1.2), we let $r_{1}=6, r_{2}=r_{3}=1, a_{11}=a_{12}=a_{21}=1$, $a_{22}=3, a_{23}=a_{32}=a_{33}=1, d_{1}=1, d_{2}=2, d_{3}=1, \tau_{1}=\tau_{2}=0.1$. Then system (1.2) has four steady states $E_{0}(0,0,0), E_{1}(6,0,0), E_{2}(4.75,1.25,0)$ and $E^{*}(4.8,1.2,0.2)$. It is easy to see that $a_{11} a_{22} a_{33}-a_{11} a_{23} a_{32}-a_{12} a_{21} a_{33}=1>0$ and (H2) holds. By Theorem 1, we know that the positive equilibrium $E^{*}$ of system (1.2) is globally stable (see Fig. 1).

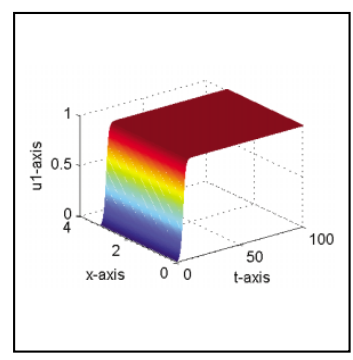

(a)

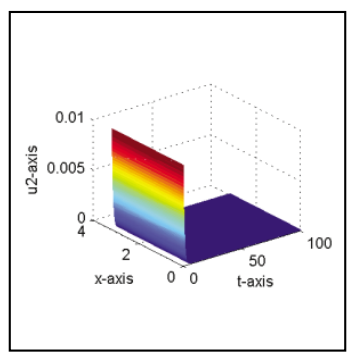

(b)

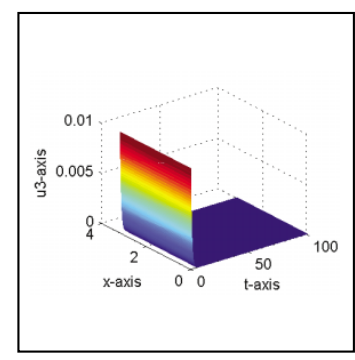

(c)

Figure 2. The time dependent solution found by numerical integration with $r_{1}=r_{2}=r_{3}=1, a_{11}=a_{12}=a_{21}=1, a_{22}=3, a_{23}=a_{32}=a_{33}=1, d_{1}=1, d_{2}=2$, $d_{3}=1, \tau_{1}=\tau_{2}=0.1 ; \phi_{i}(x, 0)=0.01(i=1,2,3) ; \partial u_{i} / \partial x=0(i=1,2,3), t \geq 0, x=0, \pi$.

Example 2. In system (1.2), we let $r_{1}=r_{2}=r_{3}=1, a_{11}=a_{12}=a_{21}=1$, $a_{22}=3, a_{23}=a_{32}=a_{33}=1, d_{1}=1, d_{2}=2, d_{3}=1, \tau_{1}=\tau_{2}=0.1$. Then system (1.2) has two steady states $E_{0}(0,0,0), E_{1}(1,0,0)$. It is easy to see that $a_{11} a_{22}-a_{12} a_{21}=2>0, a_{21} r_{1}=a_{11} r_{2}$. By Theorem 2, we know that the equilibrium $E_{1}$ of system (1.2) is globally stable (see Fig. 2). 


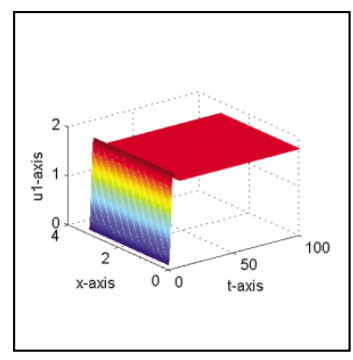

(a)

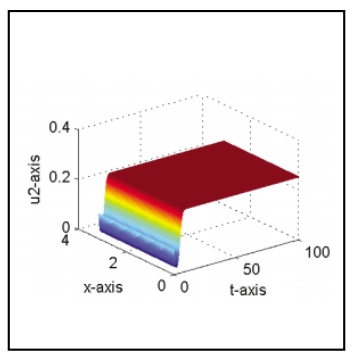

(b)

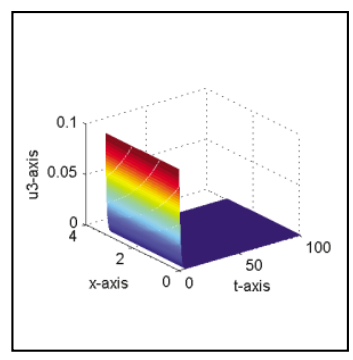

(c)

Figure 3. The time dependent solution found by numerical integration with $r_{1}=2$, $r_{2}=r_{3}=1, a_{11}=a_{12}=a_{21}=1, a_{22}=3, a_{23}=a_{32}=a_{33}=1, d_{1}=1, d_{2}=2, d_{3}=1$, $\tau_{1}=\tau_{2}=0.1 ; \phi_{i}(x, 0)=0.1(i=1,2,3) ; \partial u_{i} / \partial x=0(i=1,2,3), t \geq 0, x=0, \pi$.

Example 3. In system (1.2), we let $r_{1}=2, r_{2}=r_{3}=1, a_{11}=a_{12}=a_{21}=1$, $a_{22}=3, a_{23}=a_{32}=a_{33}=1, d_{1}=1, d_{2}=2, d_{3}=1, \tau_{1}=\tau_{2}=0.1$. Then system (1.2) has three steady states $E_{0}(0,0,0), E_{1}(2,0,0), E_{2}(1.75,0.25,0)$. It is easy to see that $a_{11} a_{22} a_{33}-a_{11} a_{23} a_{32}-a_{12} a_{21} a_{33}=1>0, a_{21} r_{1}-a_{11} r_{2}=$ $1>0, a_{21} a_{32} r_{1}-a_{11} a_{32} r_{2}-a_{11} a_{22} r_{3}-a_{12} a_{21} r_{3}=-3<0$. By Theorem 3 , we know that the equilibrium $E_{2}$ of system (1.2) is globally stable (see Fig. 3).

\section{References}

[1] N.F. Britton. Spatial structures and periodic travelling waves in an integrodifferential reaction-diffusion population model. SIAM J. Appl. Math., 50:1663-1688, 1990. Doi: $10.1137 / 0150099$.

[2] C. Cavaterra and M. Grasselli. Asymptotic behavior of population dynamics models with nonlocal distributed delays. Discrete Contin. Dyn. Syst., 22:861883, 2008. Doi:10.3934/dcds.2008.22.861.

[3] Y. Chen, J. Yu and C. Sun. Stability and hopf bifurcation analysis in a threelevel food chain system with delay. Chaos, Solitons $\mathcal{E}$ Fractals, 31:683-694, 2007. Doi:10.1016/j.chaos.2005.10.020.

[4] S.A. Gourley and N.F. Britton. A predator-prey reaction-diffusion system with nonlocal effects. J. Math. Biol., 34:297-333, 1996. Doi:10.1007/BF00160498.

[5] S.A. Gourley and S. Ruan. Convergence and travelling fronts in functional equations with nonlocal terms: a competition model. SIAM J. Math. Anal., 35:806822, 2003. Doi:10.1137/S003614100139991.

[6] S.A. Gourley and J.W.H. So. Dynamics of a food-limited population model incorporating nonlocal delays on a finite domain. J. Math. Biol., 44:49-78, 2002. Doi: $10.1007 / \mathrm{s} 002850100109$.

[7] M. Grasselli and D. Pražák. Exponential attractors for a class of reactiondiffusion problems with time delays. J. Evol. Equ., 7:649-667, 2007. Doi:10.1007/s00028-007-0326-7.

[8] Z.G. Lin. Time delayed parabolic system in the three species predator-prey model. Acta. Math. Sin., 47:559-568, 2004. 
[9] C.V. Pao. Global asymptotic stability of Lotka-Volterra three species reactiondiffusion systems with time delays. J. Math. Anal. Appl., 281:186-204, 2003. Doi:10.1016/S0022-247X(03)00033-7.

[10] H.R. Thieme and X.Q. Zhao. A non-local delayed and diffusive predator-prey model. Nonlinear Anal. RWA, 2:145-160, 2001. Doi:10.1016/S0362-546X(00)00112-7.

[11] Y.M. Wang. Global asymptotic stability of three species Lotka-Volterra models with diffusion and time delays. Appl. Math. Comput., 195:34-48, 2008. Doi:10.1016/j.amc.2007.04.068.

[12] Z.C. Wang and W.T. Li. Monotone travelling fronts of a food-limited population model with nonlocal delay. Nonlinear Anal. RWA, 8:699-712, 2007. Doi:10.1016/j.nonrwa.2006.03.001.

[13] Z.C. Wang, W.T. Li and S. Ruan. Travelling wave fronts of reaction-diffusion systems with spatio-temporal delays. J. Differential Equations, 222:185-232, 2006. Doi:10.1016/j.jde.2005.08.010.

[14] R. Xu. A reaction-diffusion predator-prey model with stage structure and nonlocal delay. Appl. Math. Comput., 175:984-1006, 2006. Doi:10.1016/j.amc.2005.08.014.

[15] R. Xu, M.A.J. Chaplain and F.A. Davidson. Periodic solutions of a three-species Lotka-Volterra food-chain model with time delays. Math. Comput. Model., 40:823-847, 2004. Doi:10.1016/j.mcm.2004.10.011.

[16] R. Xu, L. Chen and F. Hao. Periodic solutions of a discrete time Lotka-Volterra type food-chain model with time delays. Appl. Math. Comput., 171:91-103, 2005. Doi:10.1016/j.amc.2005.01.027.

[17] R. Xu and Z. Ma. Global stability of a reaction-diffusion predatorprey model with a nonlocal delay. Math. Comput. Model., 2009. Doi:10.1016/j.mcm.2009.02.011.

[18] G.B. Zhang, W.T. Li and G. Lin. Traveling waves in delayed predator-prey systems with nonlocal diffusion and stage structure. Math. Comput. Model., 49:1021-1029, 2009. Doi:10.1016/j.mcm.2008.09.007.

[19] J.M. Zhang and Y.H. Peng. Travelling waves of the diffusive Nicholson's blowflies equation with strong genetric delay kernel and non-local effect. Nonlinear Anal., 68:1263-1270, 2008. Doi:10.1016/j.na.2006.12.019. 